\title{
Hydrogen excretion in pediatric lactose malabsorbers: relation to symptoms and the dose of lactose
}

Katarzyna Pawłowska ${ }^{1}$, Rafał Seredyński ${ }^{2}$, Wioleta Umławska ${ }^{3}$, Barbara Iwańczak ${ }^{1}$

\begin{abstract}
${ }^{1} 2^{\text {nd }}$ Department and Clinic of Pediatrics, Gastroenterology and Nutrition, Medical University of Wroclaw, Wroclaw, Poland

${ }^{2}$ Department of Physical Chemistry of Microorganisms, Institute of Genetics and Microbiology, University of Wroclaw, Wroclaw, Poland

${ }^{3}$ Department of Human Biology, University of Wroclaw, Wroclaw, Poland
\end{abstract}

Submitted: 8 August 2015

Accepted: 19 October 2015

Arch Med Sci 2018; 14, 1: 88-93

DOI: $10.5114 /$ aoms.2016.57884

Copyright $\odot 2016$ Termedia \& Banach

\section{Abstract}

Introduction: Lactose malabsorption arises from lactase deficiency and may lead to lactose intolerance - gastrointestinal symptoms after lactose ingestion. Occurrence and severity of the symptoms are influenced by many factors, including the dose of lactose and the intensity of its colonic fermentation to short chain fatty acids and gases.

Material and methods: The hydrogen breath test (HBT) after $30 \mathrm{~g}$ or $50 \mathrm{~g}$ of lactose was performed in 387 children. Further analysis included children who had a positive HBT result. The HBT parameters were net hydrogen concentration in each breath and total net hydrogen concentration during the HBT. The time of the first hydrogen rise was also calculated. HBT parameters were analyzed according to symptoms occurrence (lack or present), symptoms severity (lack, moderate or severe) and the dose of lactose (30 g or $50 \mathrm{~g}$ ).

Results: One hundred and six children (12.1 years, 46 boys) had a positive HBT result. Symptoms occurrence was positively related to net hydrogen concentration at $30 \mathrm{~min}, 60 \mathrm{~min}$ and $90 \mathrm{~min}$ ( $p<0.001$ at each time point), as well as to the total net hydrogen concentration $(p<0.001)$. There were no differences in hydrogen excretion between subjects with moderate and severe symptoms after lactose ingestion. Symptoms were more frequent in subjects given $50 \mathrm{~g}$ of lactose than in those given $30 \mathrm{~g}$ of lactose (79\% vs. $47 \%, p=0.003)$. In both dose groups symptoms occurrence was related to hydrogen excretion.

Conclusions: Symptoms occurrence is closely related to hydrogen excretion and to the dose of ingested lactose.

Key words: children; gastrointestinal diseases, hydrogen breath test, lactose intolerance, lactose malabsorption.

\section{Introduction}

Deficiency of digestive enzymes may result in various health complications related to malnutrition [1]. Calcium deficit, which may arise from the difficulties in milk ingestion, may be linked to osteoporosis, type 2 diabetes, metabolic syndrome and cardiovascular disease [2, 3]. The key enzyme involved in the digestion of milk sugar, lactose, is lactase ( $\beta$-galactosidase). When lactase deficiency occurs, undigested lactose enters the colon, where it is hydrolyzed to glucose and galactose molecules by the bacterial enzyme $\beta$-galactosidase. Subsequently, these monosaccharides are fer-

\author{
Corresponding author: \\ Katarzyna Pawłowska \\ $2^{\text {nd }}$ Department \\ and Clinic of Pediatrics, \\ Gastroenterology \\ and Nutrition \\ Medical University \\ of Wroclaw \\ 50/52 M. Curie-Skłodowskiej St \\ 50-369 Wroclaw, Poland \\ Phone: +4871770 3045 \\ Fax: +48 717703046 \\ E-mail: pawlowska.katarzyna@ \\ icloud.com
}


mented to gases (hydrogen, methane and carbon dioxide) and short chain fatty acids (SCFAs). This condition is called lactose malabsorption and may result in gastrointestinal symptoms [4].

In clinical practice, lactose malabsorption is diagnosed with the hydrogen breath test (HBT), the examination consisting in breath hydrogen measurement after an oral lactose load. Although the HBT is used to detect the extent to which maldigested lactose is fermented and absorbed in the colon, it does not determine the actual activity of lactase [5]. The weak relationship between lactose digestion degree and total breath hydrogen after lactose ingestion is explained by colonic factors, which influence lactose fermentation intensity [6]. The activity of colonic microflora seems to be an important factor determining hydrogen content in breath during HBT, as well as symptoms occurrence and their severity after lactose ingestion $[4,6]$. Studies showed that only some lactose malabsorbers (LM) developed gastrointestinal symptoms after lactose ingestion, known as lactose intolerance [5, 6]. Abdominal pain, flatulence, cramps, bloating and nausea are associated with gas production, whereas loose stool or diarrhea are linked to osmotic load due to the accumulation of unfermented sugar in the colon [4].

The aim of the present study was to evaluate the influence of hydrogen excretion during a standard 90 min HBT with $30 \mathrm{~g}$ or $50 \mathrm{~g}$ of lactose on symptoms occurrence and severity in pediatric $L M$.

\section{Material and methods}

The hydrogen breath test was performed in 387 children diagnosed and treated in $2^{\text {nd }}$ Department and Clinic of Pediatrics, Gastroenterology and Nutrition (Wroclaw, Poland) in the years 2010-2013. Children underwent the HBT after an overnight fast and were asked to brush their teeth before the examination. Hydrogen content in five breaths was evaluated using the Gastro+ Gastrolyser (Bedfont Scientific, Ltd.) - one before and four after a test meal (15 min, $30 \mathrm{~min}, 60 \mathrm{~min}$, $90 \mathrm{~min})$. The test meal was lactose monohydrate (Pharma Cosmetic, Inc.), dissolved in $250 \mathrm{ml}$ of water. The dose of lactose was body weight-dependent - children up to $30 \mathrm{~kg}$ were given $30 \mathrm{~g}$ of lactose, while children over $30 \mathrm{~kg}$ were given $50 \mathrm{~g}$ of lactose. An increment of hydrogen by 20 ppm above the basal breath, in at least one breath, indicated a positive HBT result. Children with a positive HBT result were described as LM. During the study, children (and/or their caregivers) were asked to report gastrointestinal symptoms such as abdominal pain, cramping, bloating, flatulence, nausea and loose stool/diarrhea. Children with one or more symptoms after lactose ingestion were identified as lactose intolerants (LI), while those without symptoms were identified as lactose tolerants (LT). Abdominal pain, cramping, bloating, flatulence and nausea were defined as moderate symptoms, while severe symptoms were associated with one loose stool or diarrhea.

Analyses involved only children with a positive HBT result who met the inclusion criteria: (1) had no gastrointestinal disease or had one of the following - upper gastrointestinal tract disease (gastro-esophageal reflux disease or gastric ulcer disease), lower gastrointestinal tract disease (malabsorption syndrome or inflammatory bowel disease) or functional gastrointestinal disorder (functional constipation or irritable bowel syndrome); (2) had a basal breath hydrogen level lower than 20 ppm; (3) had no chronic pulmonary diseases or cow's milk allergy; (4) had no antibiotic treatment during the last month.

The HBT data collection included five parameters: hydrogen concentration in each breath after lactose ingestion minus basal hydrogen concentration (net $\mathrm{H}_{2}$ concentration at $15 \mathrm{~min}, 30 \mathrm{~min}, 60 \mathrm{~min}$ and $90 \mathrm{~min}$ ) and the sum of net $\mathrm{H}_{2}$ concentrations from 15 min to 90 min (total net $\mathrm{H}_{2}$ concentration). Mean values of each breath were used to estimate the time of the first rise of hydrogen concentration by 20 ppm using a linear regression equation.

The protocol of the study was approved by the local Ethic Committee of Medical University of Wroclaw. Informed consent was obtained from children's caregivers and children $\geq 16$ years of age.

\section{Statistical analysis}

Hydrogen concentrations were presented as mean and standard error of the mean (SEM). The distribution of the data was tested using the Kolmogorov-Smirnov test. Differences in HBT parameters between multiple groups were assessed using univariate ANOVA (parametric) or the Kruskal-Wallis test (non-parametric) with the Scheffe test (parametric) or Mann-Whitney $U$ test (non-parametric) as a post-hoc test. Two unpaired groups were compared with Student's $t$ test (parametric) or Mann-Whitney $U$ test (nonparametric). Qualitative data were tested using Pearson's $\chi^{2}$ test. A $p$-value below 0.05 was considered significant. All statistical analyses were performed using Statistica 10 software (StatSoft, Inc.). Time of the first rise of hydrogen concentration was calculated using Excel 2011 software (Microsoft, Inc.).

\section{Results}

One hundred and six children (12.1 years, 46 boys) were selected for the study from 387 children who underwent the HBT (Table I). Symptoms occurrence was positively related to net $\mathrm{H}_{2}$ concentration at $30 \mathrm{~min}, 60 \mathrm{~min}$ and $90 \mathrm{~min}$, as well 
Table I. Characteristics of subjects

\begin{tabular}{|c|c|c|c|}
\hline Diagnosis & Subjects, $n(\%)$ & Age (min.-max.) [years] & Males, $n(\%)$ \\
\hline Healthy & $35(33.0)$ & $12.17(4.29-17.56)$ & $17(48.6)$ \\
\hline Upper gastrointestinal tract disease: & $26(24.5)$ & $11.98(2.86-17.98)$ & $13(50.0)$ \\
\hline Gastro-esophageal reflux disease & 14 & $11.68(2.86-17.98)$ & $6(42.9)$ \\
\hline Peptic ulcers & 12 & $12.33(6.08-17.78)$ & $7(58.3)$ \\
\hline Lower gastrointestinal tract disease: & $25(23.6)$ & $12.06(5.27-17.90)$ & $11(44.0)$ \\
\hline Malabsorption syndromes & 15 & $10.94(5.27-16.75)$ & $6(40.0)$ \\
\hline Inflammatory bowel disease & 10 & $13.74(8.59-17.90)$ & $5(50.0)$ \\
\hline Functional gastrointestinal disorder: & $20(18.9)$ & $12.13(4.17-17.99)$ & $5(25.0)$ \\
\hline Constipation & 11 & $10.30(4.17-16.19)$ & $1(9.1)$ \\
\hline Irritable bowel syndrome & 9 & $14.36(9.25-17.99)$ & $4(44.4)$ \\
\hline Total & $106(100.0)$ & $12.08(2.86-17.99)$ & $46(43.4)$ \\
\hline
\end{tabular}

Table II. Statistical significances of differences in $\mathrm{H}_{2}$ parameters between symptom groups ( $p$-values according to $t$ test, ANOVA and Scheffe test as post-hoc, or their nonparametric counterparts)

\begin{tabular}{|lccccc|}
\hline $\begin{array}{l}\text { Net } \mathrm{H}_{2} \\
\text { concentration }\end{array}$ & Lack - present & $\begin{array}{c}\text { Lack - moderate } \\
- \text { severe }\end{array}$ & $\begin{array}{c}\text { Lack } \\
- \text { moderate }\end{array}$ & $\begin{array}{c}\text { Lack } \\
- \text { severe }\end{array}$ & $\begin{array}{c}\text { Moderate } \\
- \text { severe }\end{array}$ \\
\hline $15 \mathrm{~min}$ & 0.072 & 0.088 & - & - & - \\
\hline $30 \mathrm{~min}$ & $<0.001$ & $<0.001$ & $<0.001$ & 0.002 & 0.443 \\
\hline $60 \mathrm{~min}$ & $<0.001$ & 0.004 & 0.008 & 0.031 & 0.992 \\
\hline $90 \mathrm{~min}$ & $<0.001$ & $<0.001$ & 0.001 & 0.007 & 0.974 \\
\hline Total & $<0.001$ & $<0.001$ & 0.002 & 0.005 & 0.907 \\
\hline
\end{tabular}

as to the total net $\mathrm{H}_{2}$ concentration. Children with no symptoms exhaled about 50 ppm of hydrogen less than subjects with moderate and severe symptoms (68 ppm, $119 \mathrm{ppm}$ and $121 \mathrm{ppm}$ of total net $\mathrm{H}_{2}$ concentration; $p<0.001$ according to

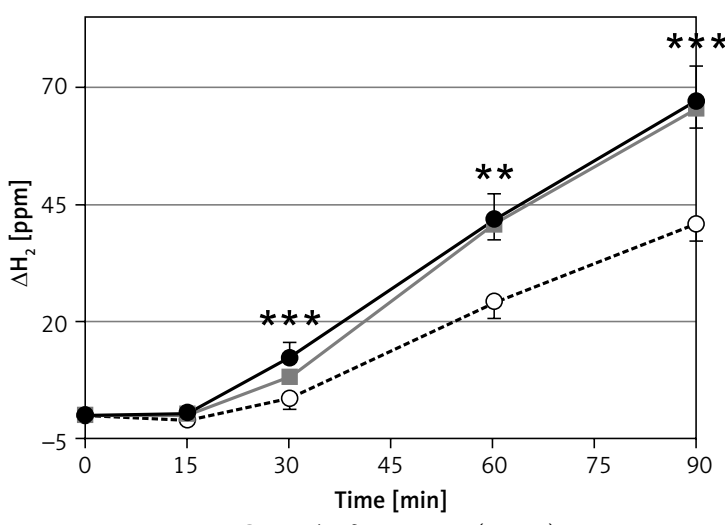

-O- Lack of symptoms $(n=33)$

Moderate symptoms $(n=52)$

$\longrightarrow$ Severe symptoms $(n=21)$

Figure 1. Net $\mathrm{H}_{2}$ concentration increment during 90 min hydrogen breath test in relation to occurrence and severity of symptoms after lactose ingestion $\left({ }^{* *} p<0.01,{ }^{* * *} p<0.001\right.$ according to univariate ANOVA or non-parametric Kruskal-Wallis test) univariate ANOVA). However, there was no difference in hydrogen excretion parameters between moderate $\mathrm{LI}$ and severe $\mathrm{LI}$ (Table II and Figure 1). Lactose tolerants achieved the first hydrogen increment in 56 min of the test $\left(R^{2}=0.992\right)$. Lactose intolerants, regardless of the symptoms severity, achieved the hydrogen increment faster than LT (in $39 \mathrm{~min}, R^{2}=0.993$ for moderate symptoms, and $37 \mathrm{~min}, R^{2}=0.999$ for severe symptoms).

Thirty-four children were given $30 \mathrm{~g}$ of lactose and 72 children were given $50 \mathrm{~g}$ of lactose. The dose of lactose was positively related to the net $\mathrm{H}_{2}$ concentration at $90 \mathrm{~min}$ and to the total net $\mathrm{H}_{2}$ concentration ( $p=0.007$ and $\left.p=0.046\right)$. These differences disappeared when analyses were performed in groups of LT and LI separately. Symptoms after lactose were significantly more frequent after $50 \mathrm{~g}$ of lactose compared to $30 \mathrm{~g}$ (79\% vs. $47 \%$, Table III). In both dose groups hydrogen excretion differed between individuals with and without symptoms. Statistically significant differences in net $\mathrm{H}_{2}$ concentration between LT and LI appeared later in children who received $30 \mathrm{~g}$ of lactose than in those given $50 \mathrm{~g}$ of lactose (Figures $2 \mathrm{~A}$ and B). Gastrointestinal disease type did not influence any HBT parameter (data not shown). 
Table III. Frequency of symptoms and dose of lactose $\left(\chi^{2}=11.834 ; p=0.003\right.$ according to Pearson's $\chi^{2}$ test)

\begin{tabular}{|lccc|}
\hline Symptoms & Dose of $\mathbf{3 0} \mathbf{g}, \boldsymbol{n}(\%)$ & Dose of $\mathbf{5 0} \mathbf{g}, \boldsymbol{n}(\%)$ & Total, $n(\%)$ \\
\hline Lack & $18(53.0)$ & $15(20.8)$ & $33(31.1)$ \\
\hline Moderate & $13(38.2)$ & $39(54.2)$ & $52(49.1)$ \\
\hline Severe & $3(8.8)$ & $18(25.0)$ & $21(19.8)$ \\
\hline Total & $34(100.0)$ & $72(100.0)$ & $106(100.0)$ \\
\hline
\end{tabular}
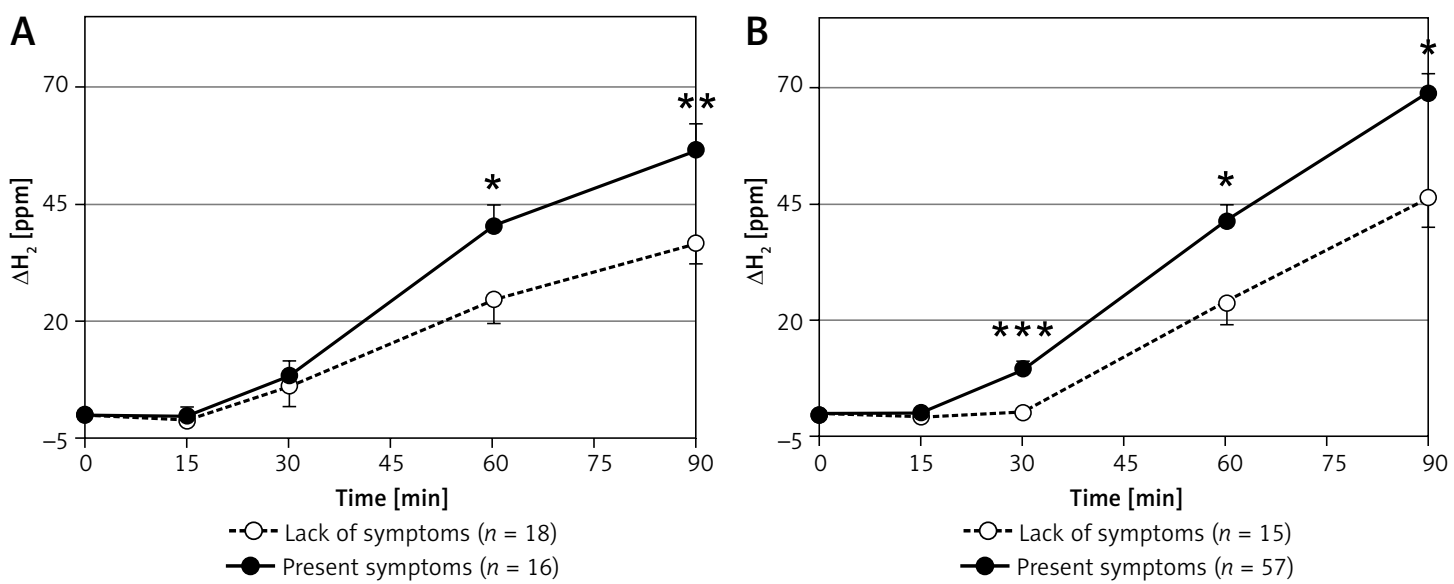

Figure 2. Net $\mathrm{H}_{2}$ concentration increment during 90 min hydrogen breath test after ingestion of $30 \mathrm{~g} \mathrm{(A)} \mathrm{or} 50 \mathrm{~g}$ (B) of lactose in relation to symptoms occurrence $\left({ }^{*} p<0.05,{ }^{* *} p<0.01\right.$, ${ }^{* * *} p<0.001$ according to unpaired Student's $t$ test or non-parametric Mann-Whitney $U$ test)

\section{Discussion}

Our study showed a significant relation of hydrogen excretion to symptoms occurrence after lactose ingestion in LM. Net $\mathrm{H}_{2}$ concentration in breath samples from $30 \mathrm{~min}$ to $90 \mathrm{~min}$ and total net $\mathrm{H}_{2}$ concentration were significantly higher in LI compared to LT. Several other studies have evidenced the relation of hydrogen excretion to symptoms occurrence after lactose ingestion. A strong positive correlation of lactose intolerance severity, expressed as total symptom score, with total hydrogen excretion was also found [7-9]. Excessive gas excretion was especially related to borborygmi and bloating [8]. According to our knowledge, only one study has found no relation between hydrogen excretion and symptoms severity [10]. All the studies contained both individuals with positive and negative HBT results, while combining these groups may inadvertently increase the relation of gas production and symptoms severity. Bianchi et al. found a high positive relation of hydrogen peak value with symptoms score in all participants, but not in a subgroup of LM [7]. Considering the above issues, all analyses in the present study were confined to individuals with positive HBT results.

Although our results showed differences in hydrogen excretion between LT and LI, no differences in HBT parameters between LI with moderate and severe symptoms were obtained. Moreover, children with lactose intolerance (regardless of symptoms severity) revealed shorter time of the first hydrogen rise compared to LT. The occurrence and severity of symptoms after lactose ingestion depend on many factors, including lactase activity, oro-cecal transit time (OCTT), lactose fermentation, colonic absorption capacity and visceral sensitivity [4]. It was demonstrated that LT had a higher degree of lactose digestion and longer OCTT than LI. However, there were no differences in these parameters between $\mathrm{LI}$ with and without diarrhea [6].

The occurrence of diarrhea is a result of high osmotic load due to accumulation of sugar in the colon. On one hand, lactose fermentation to SCFAs and gases is an important mechanism reducing osmotic load. However, excessive SCFA production reduces intraluminal $\mathrm{pH}$, which inhibits bacterial fermentation of lactose [11]. Our subjects with moderate and severe symptoms revealed similar levels of hydrogen excretion; thus symptoms severity cannot be explained by the mentioned pattern. The intensity of symptoms may be traced to the buffer capacity of the colon, rather than to the net hydrogen production. On the other hand, hydrogen content in the breath is not proportional to its production in the gastrointestinal tract. At low intestinal hydrogen production, $65 \%$ of this gas is removed by the respiratory tract, but at high hydrogen production only $25 \%$ of this gas is exhaled [12]. It is plausible that intestinal production of hydrogen is significantly different in moderate LI and severe LI. 
Our study showed that LT achieved the first hydrogen rise about 17-19 min later than LI with moderate and severe symptoms. When using lactulose, an indigestible sugar fermenting in the colon as lactose, this time may be connected with OCTT or small intestinal bacterial overgrowth. It has been demonstrated that hydrogen rise time is negatively related to abdominal pain after lactulose ingestion, and tends to be shorter in individuals with diarrhea, compared to individuals without diarrhea [13]. It was found that healthy $L M$, but tolerant, had longer OCTT than LI, and it was related to the lactose digestion degree [6]. However, another study showed similar OCTT in LM and $\mathrm{LI}$ (59 and 58 min respectively) [10].

In the present study two different doses of lactose were used, depending on patients' body weight. The dose of $50 \mathrm{~g}$ of lactose was associated with a higher incidence of symptoms compared to the dose of $30 \mathrm{~g}$. Dose of lactose is positively related to symptoms occurrence, severity and their number during HBT [9]. It has been reported by many authors that $50 \mathrm{~g}$ of the sugar (an equivalent of $1 \mathrm{~L}$ of milk) is a highly non-physiological dose, especially in countries with high prevalence of primary hypolactasia $[14,15]$. A dose of $25 \mathrm{~g}$ of lactose was suggested as an adequate dose to diagnose lactose malabsorption with reduced gastrointestinal symptoms [15]. Our study demonstrated that children who were given $30 \mathrm{~g}$ of the sugar exhaled significantly less hydrogen in 90 min of the HBT, compared to children given the higher dose. However, the distinctions disappeared when our subjects were divided additionally into symptom groups (lack or presence). Hydrogen excretion after a lower dose of lactose differed between LT and LI from 60 min of the HBT, whereas after a higher dose of lactose it did so from $30 \mathrm{~min}$. Other studies also showed that symptoms occurrence may be associated with excessive gas production only after $50 \mathrm{~g}$, and not after $25 \mathrm{~g}$, of lactose [14].

Our study was conducted on healthy children and children with various gastrointestinal diseases. We found no differences in net $\mathrm{H}_{2}$ concentration at any time of HBT, nor in total net $\mathrm{H}_{2}$ concentration, between studied groups. This allowed us to merge the mentioned groups, in order to analyze HBT parameters regardless of diagnosis. A few other studies also found no differences in hydrogen excretion after lactose between healthy subjects and those with IBS, in spite of a higher frequency of lactose intolerance in patients $[8,9]$. However, there is lack of studies presenting HBT parameters in other gastrointestinal diseases such as inflammatory bowel diseases and malabsorption syndromes.

The limitation of our study was the short duration of the HBT (90 min), which might exclude subjects with a slow hydrogen rise in the breath. It is assumed that HBT should not be shorter than 120-180 min, which gives high sensitivity and specificity [15-17]. It was shown that the first rise of hydrogen may appear between 15 and $165 \mathrm{~min}$ of the HBT after ingestion of $50 \mathrm{~g}$ of lactose, but most LM achieve their 20 ppm rise by 120 min of the test [17]. However, there are studies indicating a shorter time of the first hydrogen rise in $L M$ $[16,18]$. The second limitation of the present study was that only hydrogen was measured, whereas some individuals may exhale also or only methane. There is only one study where methane measurement has been included. It was found that excretion of both hydrogen and methane significantly influences symptoms severity, but total methane concentration is not related to ailments [7].

In conclusion, based on the presented results we conclude that only symptoms occurrence, but not their severity, is related to hydrogen excretion during the HBT. The dose of lactose positively influences symptoms occurrence and severity in LM. Breath hydrogen excretion after lactose is not affected by gastrointestinal disease type.

\section{Conflict of interest}

The authors declare no conflict of interest.

\section{References}

1. Monajemzadeh M, Ashtiani MTH, Sadrian E. Variation in plasma leptin levels in young Iranian children with cystic fibrosis. Arch Med Sci 2013; 9: 883-7.

2. Poursafa P, Kelishadi R, Amin MM, Hashemi M, Amin M. First report on the association of drinking water hardness and endothelial function in children and adolescents. Arch Med Sci 2014; 10: 746-51.

3. Pereira PC. Milk nutritional composition and its role in human health. Nutrition 2014; 30: 619-27.

4. He T, Venema K, Priebe MG, Welling GW, Brummer RJM, Vonk RJ. The role of colonic metabolism in lactose intolerance. Eur J Clin Invest 2008; 38: 541-7.

5. Perets TT, Shporn E, Aizic S, et al. A diagnostic approach to patients with suspected lactose malabsorption. Dig Dis Sci 2013; 59: 1012-6.

6. Vonk RJ, Priebe MG, Koetse HA, et al. Lactose intolerance: analysis of underlying factors. Eur J Clin Invest 2003; 33: 70-5.

7. Bianchi M, Tarquini M, Koch M, Capurso L. Correlation between symptoms and gas production in lactose intolerant subjects. Can symptoms predict a diagnosis of lactose intolerance? J Nutr Environ Med 2003; 13: 5-11.

8. Zhu Y, Zheng X, Cong Y, et al. Bloating and distention in irritable bowel syndrome: the role of gas production and visceral sensation after lactose ingestion in a population with lactase deficiency. Am J Gastroenterol 2013; 108: 1516-25.

9. Yang J, Deng Y, Chu H, et al. Prevalence and presentation of lactose intolerance and effects on dairy product intake in healthy subjects and patients with irritable bowel syndrome. Clin Gasrtoenterol Hepatol 2013; 11: 262-8. 
10. Zhao J, Fox $M$, Cong Y, et al. Lactose intolerance in patients with chronic functional diarrhea: the role of small intestinal bacterial overgrowth. Aliment Pharmacol Ther 2010; 31: 892-900.

11. Holtung K, Clausen MR, Hove H, Christiansen Mortensen BP. The colon in carbohydrate malabsorption: short-chain fatty acids, $\mathrm{pH}$, and osmotic diarrhoea. Scand J Gastroenterol 1992; 27: 545-52.

12. Carbonero F, Benefiel AC, Gaskins HR. Contributions of the microbial hydrogen economy to colonic homeostasis. Nat Rev Gastroenterol Hepatol 2012; 9: 504-18.

13. Madrid AM, Landskron G, Klapp G, Reyes A, Pizarro C, Defilippi C. Lactulose hydrogen breath test and functional symptoms in pediatric patients. Dig Dis Sci 2012; 57: 1330-5.

14. Ghoshal UC, Kumar S, Misra A, Mittal B. Lactose malabsorption diagnosed by $50-\mathrm{g}$ dose is inferior to assess clinical intolerance and to predict response to milk withdrawal than 25-g dose in an endemic area. J Gastroenterol Hepatol 2013; 28: 1462-8.

15. Rana SV, Malik A. Hydrogen breath tests in gastrointestinal diseases. Ind J Clin Biochem 2014; 29: 398-405.

16. Casellas F, Malagelada JR. Applicability of short hydrogen breath test for screening of lactose malabsorption. Dig Dis Sci 2003; 48: 1333-8.

17. Bate JP, Irving PM, Barrett JS, Gibson PR. Benefits of breath hydrogen testing after lactulose administration in analysing carbohydrate malabsorption. Eur J Gastroenterol Hepatol 2010; 22: 318-26.

18. Dabritz J, Muhlbauer M, Domagk D, et al. Significance of hydrogen breath tests in children with suspected carbohydrate malabsorption. BMC Pediatrics 2014; 14: 59. 